\title{
A Systematic Approach to the Comparison of Roles in the Software Development Processes
}

\author{
Murat Yilmaz ${ }^{1}$, Rory V. O'Connor ${ }^{2}$ and Paul Clarke ${ }^{1}$ \\ 1 Lero Graduate School in Software Engineering, Dublin City University, Ireland \\ ${ }^{2}$ Lero, the Irish Software Engineering Research Centre, Dublin City University \\ \{murat.yilmaz,roconnor,pclarke\}@computing.dcu.ie
}

\begin{abstract}
The vision of building a successful software product requires teams of individuals equipped with a wide range of social and technical skills. Furthermore, by combining these skills with appropriate job roles, we should be able to improve the productivity of a software organization. In order to identify and compare different roles in software development activities, we conduct a systematic comparison of software development models, covering traditional approaches through to agile techniques. To compare the roles in the literature with industrial software landscapes, we use data from a survey conducted on 266 software practitioners to ascertain job roles in two middle size software companies, one of which uses traditional methods and in particular ISO/IEC 12207 for managing their software development activities while other uses a tailored agile methodology. In light of our interviews, we found that based on project specific needs, the roles used in industry vary significantly from the roles defined in literature.
\end{abstract}

\section{Introduction}

Software development is a complex socio-technical activity, which relies on teams of individuals working harmoniously. Therefore, individuals should be able to cope with challenges embedded in software development tasks. These tasks, however, should be performed as teamwork to accomplish a particular contract with stakeholders [1]. During these activities, the socio-technical skills of individuals are an important consideration when forming teams. As mentioned in every software development methodology, there are job roles for individuals to be assigned, and there is always a type of process it follows to progress [2]. A role, on the other hand, is a series of expectations from an individual based on the team-based activities that are defined in a social context or a situation. To achieve successful results, these individuals should be selected for a appropriate role based on the skills mentioned, which also usually creates a kind of dependency on their tasks.

While the development process involves lots of social interactions, and participants with overlapping responsibilities, defining proper interactions among these roles are important for software development activities so that the participants 
will know what to do and which parts of a task fall under their responsibility. However, without portraying the needs of these roles in a social structure, definition of these roles may not be enough for organizing a software team and particularly for orchestrating a software organization as a whole.

There are several software development roles that are defined by traditional development approaches. The agile perspective somehow changed the flow of information in a software company, which causes alterations in several roles or job titles previously defined in traditional software development. This realignment has weakened some of the traditional roles to some extent: therefore even some practitioners think that agile reduces the ability of managers to command their teams [3].

In this paper, we constitute a systematic comparison framework based on actualized roles and defined roles in the software development processes. We formalize our research question as: "In practice, do software development roles differ from the role definitions provided by the software development process methodologies?" To this end, we review the literature to single out the set of defined roles for the selected software development processes and systematically compare them with the roles that are used in industrial settings. Based on a case study with two middle size software companies, we first use the data collected on our surveys to understand the working roles or titles in an industrial software organization, and secondly we interview software practitioners to validate our results.

The remainder of this paper is structured as follows: In section two, we introduce our research viewpoint, which defines our systematic approach that enables the comparison of different roles. The following section reviews the roles identified in literature for the different software development processes. The next section evaluates our approach by analyzing of data gathered from the case studies we conducted in two middle size software companies. The last section will conclude the paper with a brief summary of contributions.

\section{Research Overview}

The first part of our systematic approach starts with constructing our research goal to evaluate whether there is a significant amount of difference in previously identified roles and their actualizations especially when tailoring a role-based task assignment in software development. Next, we survey the literature for the roles for both traditional and agile methodologies that are mentioned in software development literature. We selectively chose software methodologies and processes and work on the roles that are defined by these approaches. In technical terms, we conduct a thematic content analysis (i.e. descriptive presentation of this literature review) based on roles as the units of analysis. After identifying software development roles in the literature, secondly we conduct a focus group study with one of our industrial partners, where we seek opinions about actual roles that are used in their company. We initiate the focus group conversation by using some parts on our previously conducted survey, in which we ask par- 
ticipants about their organizational roles and experience levels on that role (see figure 1). Secondly, we interview team leaders and development managers about how accurate the actualization of the job roles.

Content analysis is an organized study of characteristics found in a content of any type of communication, such as books, websites, newspapers, etc [4]. Our approach uses the content analysis technique for making interpretations to create a role selection schema based on literature of roles in software development methodologies. Based on the survey data collected previously, these roles will be systematically compared to their industrial actualizations. To this end, we first collect data from literature and consult industry about the defined roles frequently used in software engineering settings. Secondly, we conduct a focus group, where we record the session and a content analysis was performed on participants' definition of roles that are actualized in software development landscapes.

We form a number of acronyms based on the roles that are found from the literature. Here, we are making partial use of a coding mechanism to construct a role-based schema with the defined roles from the literature. The coding aims to create variables based on the roles defined in software development. It is done for easy comparison of roles by constructing a unique key for each role found from the literature. Our coding schema allows us to observe the commonalities and differences between software engineering roles. It helps us to investigate causeeffect relationships, interrelationships, and situational conditions for each role category. Here, we design several questions to seek validity for our coding in the defined categories, and analysis of identified roles from the literature.

- Is this role the same as a role in the other categories?

- Are there any duplicated role codings in a category?

- In which context do these roles emerge?

- What kind of roles have changed or evolved in emerging methods?

- Is there any observable change for other roles when a role evolved to an other form (i.e. covariance between categories)?

The objective coding [5] is a technique to review a bunch of documents for extracting and indexing the information so as to form a new perspective on representing the data. We use an objective coding scheme on the collected information of roles. This coding should be helpful for visually comparing actualized roles systematically with the ones cited in the literature. In addition, a diagram is drawn to support the development of the relationship among roles (see Figure 1).

Finally, we aim to formulate a framework for software practitioners, which enable them to select proper roles for their software development methodologies. Consequently, by using such a framework, a software practitioner may easily choose or customize the necessary roles for his or her development activities. 


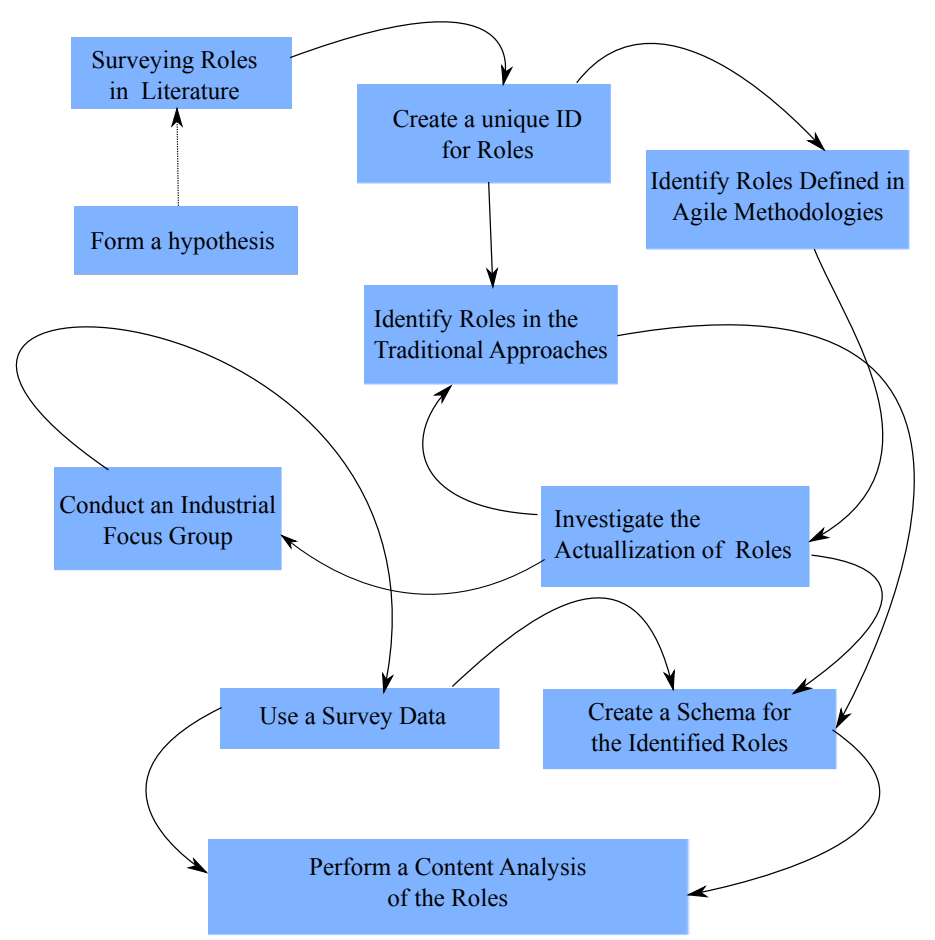

Fig. 1: Our Systematic Approach for Investigating the Roles in Software Development Environments.

\section{Roles in Software Development Processes}

Many different variants of development models and methodologies have been created. In this section, we survey the roles that are defined in the literature starting from traditional software development and working through ISO/IEC 12207, and agile methodologies such as extreme programming (XP), scrum and feature driven development (FDD).

\subsection{Roles in traditional software development}

Software engineering teams address the complex problems of software development by sharing the tasks among its members with respect to their roles. Roles are the descriptions of duties or assignments and competence for participants that are required to achieve a defined tasks and activities of software development [6]. In his essay, The Cathedral and the Bazaar, Raymond states that because of the strict roles defined in traditional software development, traditional approach is similar to building a cathedral, where a small team of people working in an isolated environment [7]. Therefore, this could be considered as a drawback because several artifacts are only visible for a limited number of individuals in this setting. 


\begin{tabular}{|l|l|l|}
\hline Code & Role Name & Primary Type of Value \\
\hline PM & Project Manager & Resource Allocation and Budgeting \\
\hline SD & Software Developer & Development Activities \\
\hline UID & User Interface Designer & Design Screen Interfaces \\
\hline DD & Database Designers & Data Modeling \\
\hline SA & Software Architects & Software Modeling \\
\hline BA & Business Analyst & Stakeholder Management \\
\hline RE & Requirement Engineer & Gathering Requirements \\
\hline SQA & Software Quality Assurance & Creating and Maintaining Quality \\
\hline SAN & System Analyst & Construction of a System \\
\hline
\end{tabular}

Table 1: Traditional Software Development Roles

Traditional roles include: Project manager who is responsible for allocation of resources, project expenditures, and responsible from the general objectives of a software project. Another typical role in the development processes is the role of a developer. A software developer is responsible for designing and maintaining the software programs, whereas a software tester is responsible for creating test plans and testing the developed programs. In many cases user interface designers (design screen interfaces), database designers (design database schema) and the software architects (design technical blueprints) are also included as a generic software practitioner category. A business analyst is not only responsible for solving the problems by regulating the connections between the business and the technical people but also for documenting several parts (e.g. requirement documents) of a software project. In addition to these roles some others can also be seen regarding several needs; e.g. requirements engineer, systems analyst, software quality assurance engineer (see Table 1) .

\begin{tabular}{|l|l|l|}
\hline Code & Role Name & Primary Type of Value \\
\hline RO & Requirements Owner & Understanding Need \\
\hline SD & System Designer & Accomplishing work \\
\hline SA & System Analysis & Reducing Risks \\
\hline VV & Validation \& Verification & Mitigating Risks \\
\hline LO & Logistics and Operations & Understanding need \\
\hline G & Glue among the subsystems & Accomplishing work, Reducing Risks \\
\hline CI & Customer Interface & Understanding the Need \\
\hline TM & Technical Manager & Technical Management \\
\hline IM & Information Manager & Knowledge Management \\
\hline PE & Process Engineer & Managing and Understanding Needs \\
\hline CO & Coordinator & Organizational Management \\
\hline CA & Classified Ads SE & Accomplishing Work (assumed) \\
\hline
\end{tabular}

Table 2: Systems Engineering Roles and their values from [8]

Sheard [9] identifies twelve roles (see Table 2) of development from system engineering viewpoint while investigating the relationship between the roles and their importance for creating a value. This work not only suggests that the value is asserted in qualitative terms and it should be quantified in further research but it also claims that it should be observed as a requested improvement within a 
product by better (i) definition of the requirements, (ii) management strategies, (iii) ways for mitigating risks, (see [8] for details).

\subsection{Roles in ISO/IEC 12207}

ISO/IEC 12207 [10] has three main groups of roles for its participants. The first group consists of the principal roles are the acquirer, who is a form of stakeholder that obtains products or services from supplier, who is an individual or another organization agree on providing a software products or services. Implementer executes development tasks, while the maintainer can be either an organization or an individual who performs the upkeep of developed software), and operator is responsible for the execution of a system [10]. The second category consists of configuration and supporting roles; the configurator is responsible for the establishment and transformation of the information needed by an individual or a group, evaluator tests and measure a software process or a product by using the data collected during the actual tasks that are performed, the auditor investigates the products and processes are compatible with the agreements, the usability specialist deals with the demands and needs of the stakeholders such as the design activities based on human factors and skills and their fulfillment [10].

\begin{tabular}{|l|l|l|}
\hline Code & Role Name & Primary Type of Value \\
\hline AC & Acquirer & Software Client or User or Product Owner \\
\hline SU & Supplier & Software Producer, Product Seller \\
\hline IMP & Implementer & Realization of Development Tasks \\
\hline MN & Maintainer & Maintain the Software \\
\hline OP & Operator & System Execution \\
\hline CON & Configurator & Accomplishing Work, Reducing Risks \\
\hline EV & Evaluator & Test \& Measure a Process or a Product \\
\hline AU & Auditor & Contract Management \\
\hline US & Usability Specialist & Problems Regarding to People Factors \\
\hline MA & Manager & Managing \\
\hline AM & Asset Manager & Managing Assets \\
\hline CM & Knowledge Manager & Knowledge Management \\
\hline RA & Reuse Administrator & Seeking for Reusable Parts \\
\hline
\end{tabular}

Table 3: Roles in ISO/IEC 12207 (adapted from $[10,11]$ )

The third group has the organizational roles, the manager identifies and manages the state of the play (i.e. condition and progression of the project) with respects to project constraints (e.g. objectives, budget, schedules), the asset manager is a type of manager deals with the management and optimization of the assets regarding to the plan he or she prepared, the knowledge manager role works on the collection of particular knowledge and skills throughout the organization and used for improvement for the products and services. The reuse program administrator seeks to find favorable or advantageous circumstances for reusable parts of a product or a service. Unlike the other two subfields of software engineering (i.e. requirements engineering and software development), domain engineer is a form responsible for designing the domain models (i.e. software models) and domain descriptions for a software system (see Table 3). 


\subsection{Roles in Extreme Programming}

According to Beck [12], the participants and their roles are as follows; Programmers are the individuals who need to have good communication and collaboration skills for both team and individual levels. They are responsible for developing, maintaining and testing the software. One of their main responsibilities is to ensure that their work is clean and lean. The technical decisions are made by programmers. Customers form the steering teams in business terms and in particular in requirement satisfaction decisions. Testers help customers to write functional test cases. Business decisions are made by customers [12]. The tracker role composes a trace and feedback mechanism in XP. The estimations, goals and iterations made by teams are controlled by a tracker, who provides feedback. The tracker is also responsible for measuring constraints such as scarce resources and delivery times versus goal evaluation. The coach is the role which is accountable for XP project who needs to understand the problems occurring during the process to instruct team members and transfer the information or sometimes experience among teams and individuals. Finally, the manager is responsible for final decisions, and also an aim of this role is to recognize problems likely occur during the development life-cycle (see table 4).

\begin{tabular}{|l|l|l|}
\hline Code & Role Name & Primary Type of Value \\
\hline PRG & Programmers & Maintaining and Testing Software \\
\hline CU & Customers & Managing Business Decisions \\
\hline TST & Testers & Helps Costumers for Functional Test Cases \\
\hline TRC & Tracker & Feedbacks and Estimations \\
\hline CO & Coach & Supervise Team \\
\hline CON & Consultant & Guides the Team for Problem Solving \\
\hline MA & Manager & Management \\
\hline
\end{tabular}

Table 4: Roles in XP (adapted from [12, 13])

\subsection{Roles in Scrum}

Schwaber and Beedle [14] single out six roles for the participants of Scrum. The Scrum Master is a type of management role specific to Scrum, who is responsible for the alignment of practices and rules as they have organized. This role interacts not only with project team but also customer and management. Its aim is to maximize productivity by practicing the agile and scrum values and monitoring the team to avoid any kind of complications. The Product Owner is the role which is responsible for exercising the project management and control activities. Additionally, this role is also responsible for transforming the product backlog into product features. Scrum Team should be considered as a self organizing structure to produce a working piece of a product, where its main goal is to achieve time targeted objectives of each sprint. The customer role will continuously evaluate the backlog items, and helps the selection for a sprint. The management role is responsible for implementing the proper standards for 
the software development process. Additionally, this role encompasses decision making activities and finalizing them at different stages of development process such as evaluating goals, gathering requirements, etc. (see Table 5).

\begin{tabular}{|l|l|l|}
\hline Code & Role Name & Primary Type of Value \\
\hline SM & Scrum Master & Managing Scrum Team \\
\hline PO & Product Owner & Product Management Decisions \\
\hline CUS & Customer & Evaluation of backlog items \\
\hline ST & Scrum Team & Organized itself for time boxed goals \\
\hline MNG & Management & Evaluate Decisions and Goals \\
\hline USR & User & Evaluate System Functionalities \\
\hline
\end{tabular}

Table 5: Roles in SCRUM (adapted from [14])

\subsection{Roles in FDD}

FDD has the most comprehensive role description with a flexibility of roles [15]. For example, an individual can play multiple roles, or either a role can be shared by multiple persons [13]. The three main categories of roles, which are: key, supporting and additional roles. The key roles are project manager, who administers the entire project and maintains the work settings of the software team, the lead software architect is the role which makes the appropriate decisions for software development, the software development manager is a role which focuses on daily activities and team negotiations during the software development activities. The lead programmer, the class owner and the domain expert are the three roles used in FDD. The supporting roles includes; manager (release), knowledge expert, build process engineer, toolsmith and system administrator. Moreover, testers, technical document expert and software deployment personnel are the other roles used in this practices [15](see table 6).

\begin{tabular}{|l|l|l|}
\hline Code & Role Name & Primary Type of Value \\
\hline PM & Project Manager & Resource Management \\
\hline LSA & Lead Software Architect & Architectural Decisions \\
\hline DEM & Development Manager & Evaluation of backlog items \\
\hline LP & Lead Programmer & Organized itself for time boxed goals \\
\hline CO & Class Owner & Form Teams for Implementing Features \\
\hline DE & Domain Expert & Inform Teams for Adequate Features \\
\hline RM & Release Manager & Managing the development process \\
\hline DM & Domain Manager & Managing Domain Experts \\
\hline LG & Language Guru & Acquiring a Knowledge on Technology \\
\hline BE & Build Engineer & Executing a Build Process \\
\hline TA & Toolsmith & Creating Utilities for project \\
\hline SYA & System Administrator & Administration of Work Systems \\
\hline TE & Testing & Verifying the Actualization of a System \\
\hline DEP & Deployer & Release of Feature Deployment \\
\hline TEW & Technical Writer & The Documentation for Users \\
\hline
\end{tabular}

Table 6: Roles in FDD (adapted from [15, 13]) 


\section{Evaluation of Roles from Industrial Settings}

As a part of a survey, we asked 266 participants from two different software companies about their roles in their applied settings in order to identify the commonality of meaning in the different roles. One of the software companies (with a staff about 400 personnel) is working in telecommunication sector, which composes solutions for large-scale e-government projects. The other company supplies turn key software solutions to telecommunications operators and mobile service providers. It has a staff of about 40 personnel. By creating a list of roles based on the roles mentioned in the literature, we conduct a focus group in one of the companies about the actualization of roles in development environments. This brings individuals together to debate about software development roles in their company and their actualizations with respect to their experiences. Next, we ask our research question to a selection of people mostly to the individuals from the management teams.

Company A is using the traditional software development approaches to define the roles: PM, SD, UID, SA, BA, SQA, where DD is embedded in SD, and $\mathrm{RE}$ role is somehow split with $\mathrm{BA}$ and $\mathrm{SD}$. The role of system analyst provides the requirement engineering processes.

Interview quotation: "During our development activities, we observe lots of overlapping roles, which sometimes hinder our ability to handle some development tasks. For example, some of our teams have key players with overlapping roles and some individuals perform more than one role by the nature of our development process. We found it interesting to have a big picture of the roles in the different software development processes."

Company A uses ISO/IEC 12207 combined with an iterative development schema and a customized role selection based on the traditional viewpoint for developing and maintaining software project. However the roles defined by ISO/IEC 12207 are not fully used to profile the personnel. Instead, they use the role names (see Table 1) that are traditionally used in software development.

Interview quotation: "We use approximately 14 out of 43 processes, 60 out of 95 activities, 180 out of 406 tasks from ISO/IEC 1220\%. We believe that assigning suitable roles to teams and individuals is very important for our success. A review of roles in different methodologies is useful from an industrial perspective. All type of roles should be visible to everyone in the company, and they should be defined in a simple language to provide a way of ensuring everyone understands them. Therefore, we are not using the role names provided by ISO/IEC 1220\%. I would say, we mostly use the classical role names you have mentioned."

According to the management team of Company A, the role of team leader should not dictate anything to teammates but communicated the vision of a 
company or a project. Therefore, maintaining a friendship and trust is more important than dictating the facts to software teams.

Interview quotation: "People usually trust other people to some extent. There are always problems, when it comes to role assignment as well as delegations based on these roles. I personally observed several situations, where improper delegation did cause lots of conflicts and tensions. I would strongly suggest that role tailoring should not be taken lightly."

Company B uses a customized agile methodology, which relies on XP and Scrum. They use agile methodology so as to cope with dynamically challenging requirements and to fulfill the request of their customer for continuous integration with small increments. They use all roles defined by scrum (i.e. SM, PO, CUS, ST, MNG, USR) and a tester role (TST) and a progress tracker (TRC) role from XP.

Interview quotation: "There is the notion of tailoring methodologies, how about the roles? It is always a problem for us to select the suitable roles for our customized methodology. Therefore, broader view of roles in software development activities are very important for us. However, just as there is no one-size-fits-all methodology for developing applications in software development, there should not be a one-size-fits-all approach to role selection."

Finally, Company B highlights the importance of face to face communication for agile landscapes, and therefore selection of suitable roles for development activities becomes more important.

Interview quotation: "The process of customization of roles is very important particularly in agile development environments. A summary with roles contained in different agile approaches is very helpful for us to see the suitable roles for our process."

\section{Conclusions}

In this paper, we highlight how roles in literature and their actualizations on industrial environments vary for both plan driven and agile methodologies. Software development is a collaborative endeavor that depends on its development methodology. However, selection of a proper methodology is not enough for achieving goals of a software organization. The evidence suggests that we should also tailor the necessary roles depending on development activities.

After analyzing the defined categories in light of the questions above, we confirmed that several roles presented in older methods are emerged with a 


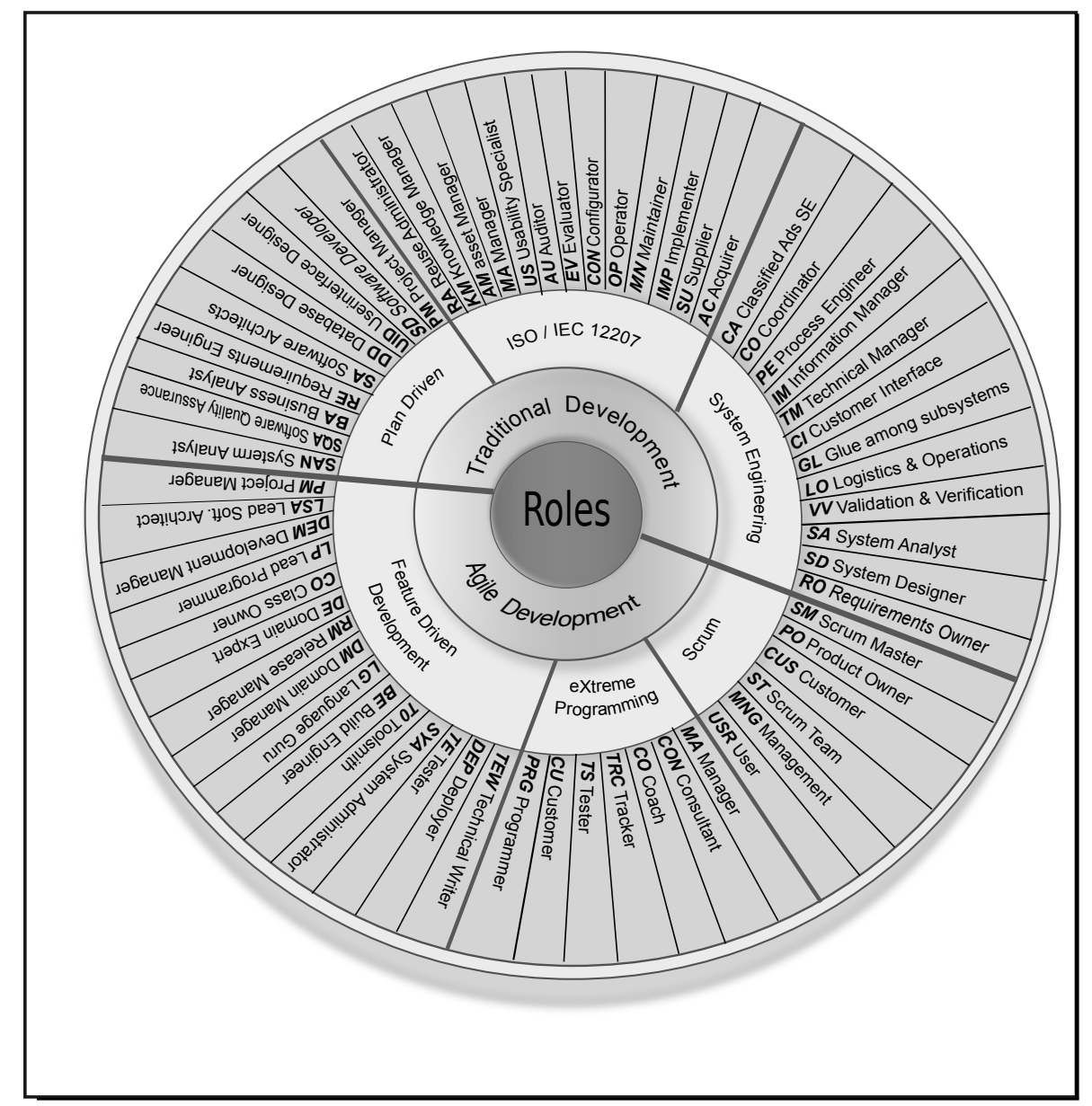

Fig. 2: A Summary of Roles Contained in the Different Approaches

different name, with similar responsibilities in newer approaches. Some of the roles, however, have their responsibilities changed while revealing in different software development organizations. Most frequently, the role definitions that an organization uses based on a domain and a set of circumstances. Our study exhibits that a role-based schema can be useful for a tailoring process of roles regarding to the organizational needs. Furthermore, we argue that a software development organization should customize their own roles suitable for their social structure, where we suggest that our role based construct (see Figure 2) will be beneficial for such activities. In other words, it enables them to select proper roles for their software development methodologies. Consequently, by using such a framework, a software teams may easily choose or customize the necessary roles based on their activities. 
Analysis of identified roles from the literature is portrayed in Figure 2. We can confirm that several roles presented in older methods are emerged with a different name with similar responsibilities in newer approaches. The roles, however, mostly have their responsibilities changed and reappeared as another form while revealing in different software development organizations. Most frequently, the role definitions that an organization uses based on a domain and a set of circumstances. Moreover, it is important to choose roles, based on the social structure of an organization and required interactions. These customized roles are found to be organizational centric, which also clearly supports the notion of separation of concerns [16].

\section{Acknowledgments}

This work is supported, in part, by Science Foundation Ireland grant number

03/CE2/I303-1 to Lero, the Irish Software Engineering Research Centre (www.lero.ie).

\section{References}

1. Humphrey, W.: Introduction to the team software process (sm). Addison-Wesley Professional (2000)

2. Zahran, S.: Software Process Improvement: Practical Guidelines for Business Success. Addison Wesley (1998)

3. Larman, C.: Agile and iterative development: a manager's guide. Addison-Wesley Professional (2004)

4. Krippendorff, K.: Content analysis: An introduction to its methodology. Sage Publications, Inc (2004)

5. Glaser, B., Strauss, A.: The discovery of grounded theory: Strategies for qualitative research. Aldine Transaction (2007)

6. Sommerville, I.: Software Engineering (9th Edition). Addison Wesley (2009)

7. Raymond, E.: The cathedral and the bazaar. Knowledge, Technology \& Policy 12 (1999) 23-49

8. Sheard, S.: The value of Twelve systems engineering roles. In: Proceedings of INCOSE, Citeseer (1996)

9. Sheard, S.: Twelve systems engineering roles. In: Proceedings of INCOSE, Citeseer (1996)

10. ISO/IEC: Amendment to ISO/IEC 12207-2008 - Systems and software engineering Software life cycle processes. (2008)

11. Acuna, S.T., Juristo, N., Moreno, A.M., Mon, A.: A Software Process Model Handbook for Incorporating People's Capabilities. Springer-Verlag (2005)

12. Beck, K.: Extreme programming explained. Addison-Wesley (2000)

13. Abrahamsson, P., Salo, O., Ronkainen, J., Warsta, J.: Agile software development methods: Review and Analysis. Volume VTT Publications 478. Technical Research Centre of Finland (2002)

14. Schwaber, K., Beedle, M.: Agile Software Development with SCRUM. Prentice Hall (2002)

15. Palmer, S.R., Felsing, J.M.: A practical guide to feature-driven development. Prentice Hall PTR (2002)

16. Parnas, D.L., Clements, P.C.: A rational design process: How and why to fake it. IEEE Trans. Softw. Eng. 12 (1986) 251-257 